Short Communication

\title{
Parallel trends in the transmission of SARS-CoV-2 and retail/ recreation and public transport mobility during non-lockdown periods
}

\author{
Bernard Cazelles $^{\mathrm{a}, \mathrm{b}, *}$, Catherine Comiskey ${ }^{\mathrm{c}}$, Benjamin Nguyen-Van-Yen ${ }^{\mathrm{b}, \mathrm{d}}$, \\ Clara Champagne ${ }^{\mathrm{e}, \mathrm{f}}$, Benjamin Roche ${ }^{\mathrm{g}}$ \\ a UMMISCO, Sorbonne Université, Paris, France \\ b Eco-Evolution Mathématique, IBENS, UMR 8197, CNRS, Ecole Normale Supérieure, Paris, France

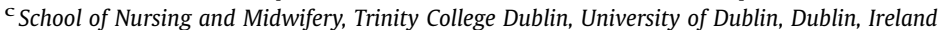 \\ d Institut Pasteur, Unité de Génétique Fonctionnelle des Maladies Infectieuses, Paris, France \\ e Swiss Tropical and Public Health Institute, Basel, Switzerland \\ ${ }^{\mathrm{f}}$ University of Basel, Basel, Switzerland \\ ${ }^{\mathrm{g}}$ MIVEGEC, IRD, CNRS and Université de Montpellier, Montpellier, France
}

\section{A R T I C L E I N F O}

\section{Article history:}

Received 15 December 2020

Received in revised form 25 January 2021

Accepted 26 January 2021

\section{Keywords:}

SARS-Cov-2

Transmission

Effective reproduction number

Mobility

\begin{abstract}
A B S T R A C T
Recent literature strongly supports the hypothesis that mobility restriction and social distancing play a crucial role in limiting the transmission of severe acute respiratory syndrome coronavirus-2 (SARS-CoV2). During the first wave of the coronavirus disease 2019 (COVID-19) pandemic, it was shown that mobility restriction reduced transmission significantly. This study found that, in the period between the first two waves of the COVID-19 pandemic, there was high positive correlation between trends in the transmission of SARS-CoV-2 and mobility. These two trends oscillated simultaneously, and increased mobility following the relaxation of lockdown rules was significantly associated with increased transmission. From a public health perspective, these results highlight the importance of tracking changes in mobility when relaxing mitigation measures in order to anticipate future changes in the spread of SARS-CoV-2.
\end{abstract}

(c) 2021 The Authors. Published by Elsevier Ltd on behalf of International Society for Infectious Diseases. This is an open access article under the CC BY-NC-ND license (http://creativecommons.org/licenses/by-nc-

nd $/ 4.0 /$ ).

The mobility of hosts and/or vectors has always influenced the transmission of disease (Tizzoni et al., 2014), and this is true for coronavirus disease 2019 (COVID-19) (Kraemer et al., 2020).

Recent literature strongly supports the hypothesis that mobility restriction and social distancing play a crucial role in limiting the transmission of severe acute respiratory syndrome coronavirus-2 (SARS-CoV-2). During the first wave of the COVID-19 pandemic, it was shown that mobility restriction reduced transmission significantly. Some studies concluded that mobility patterns were correlated with the prevalence of COVID-19, and that travel restriction reduced the transmission of SARS-CoV-2 (Badr et al., 2020; Chang et al., 2021). Other studies used changes in mobility patterns to estimate the effect of mitigation measures on the reproduction number, and showed that mobility restriction, coupled with the introduction of mitigation measures, led to a

\footnotetext{
* Corresponding author at: Eco-Evolution Mathématique, IBENS, Ecole Normale Supérieure, 46 rue d'Ulm, Paris 75230, France.

E-mail address: cazelles@bologie.ens.fr (B. Cazelles).
}

sharp decrease in transmission (Lemaitre et al., 2020; Park et al., 2020). However, these results were limited to the first wave of the COVID-19 pandemic, before and during the first lockdown period.

It remains unknown whether increased mobility following the relaxation of lockdown rules is associated with enhanced transmission of SARS-CoV-2. In addition to mobility patterns, lockdowns impact a wide range of dimensions in society. This makes it difficult to draw a causal link between mobility and viral transmission from lockdown data. To improve understanding, investigation of this association in the interval between lockdown periods was undertaken to determine if mobility data can be used as an early signal for the implementation of public health measures.

This study found strong correlation between trends in the transmission of SARS-CoV-2 and mobility in the period between the first two waves of the COVID-19 pandemic in various French regions and Ireland. The aim of this study was to compare different geographical settings with similar population sizes. Changes in the transmission of SARS-CoV-2 were quantified by computing the effective reproduction number $R_{\text {eff }}(t)$. This is defined as the average 

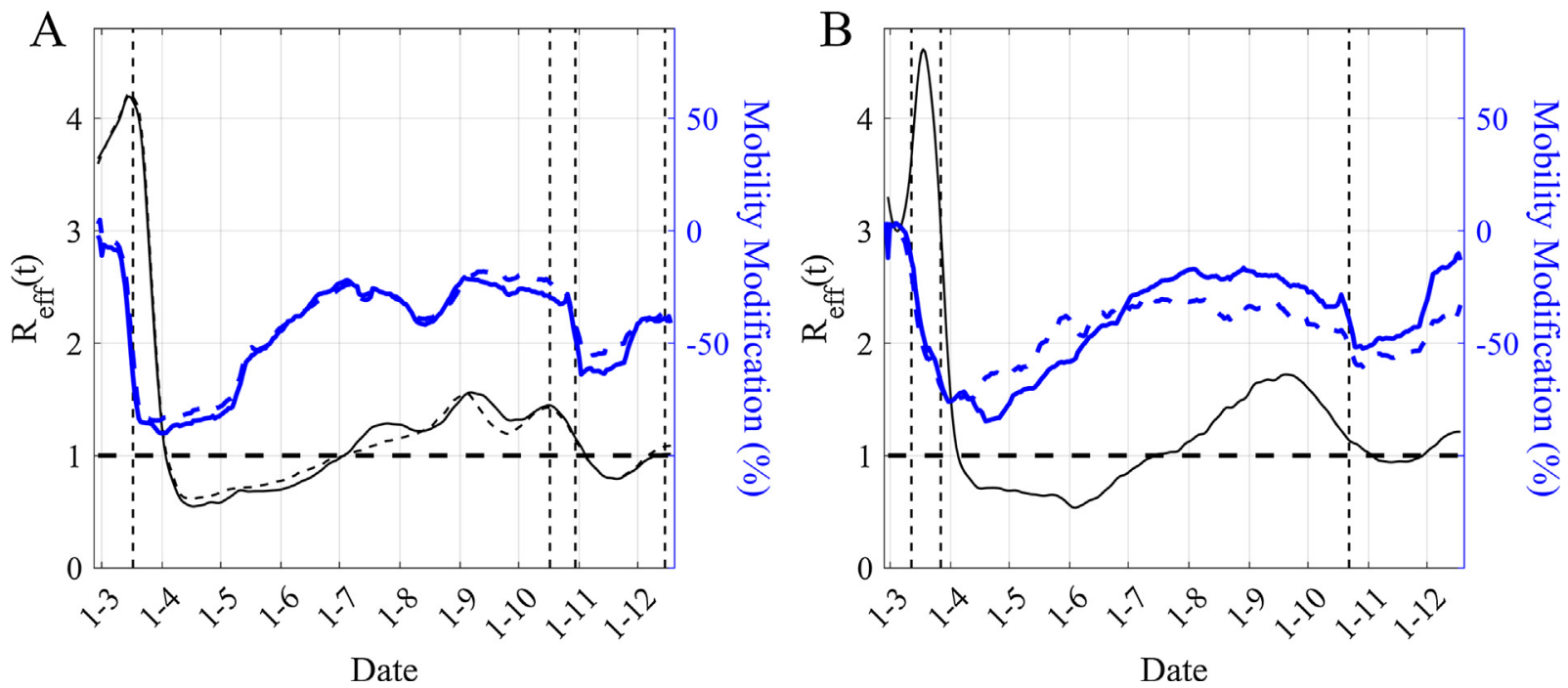

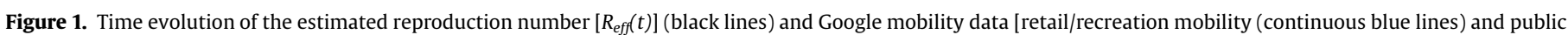

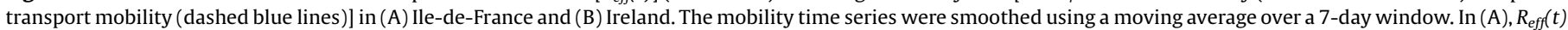

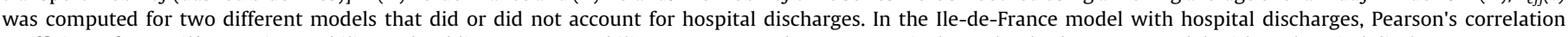

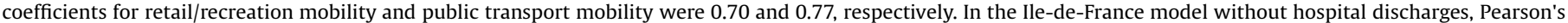

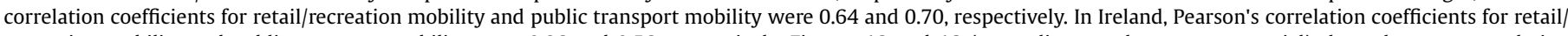

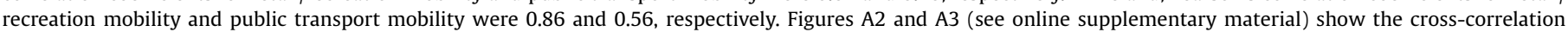

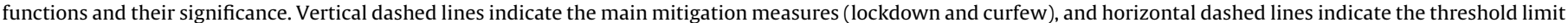
for $R_{\text {eff }}(t)$. (For interpretation of the references to color in this figure legend, the reader is referred to the web version of this article.)

number of secondary cases at time $t$ arising from a primary infected case. $R_{\text {eff }}(t)$ is an important metric to measure timevarying transmissibility and assess the effectiveness of different interventions. The classical statistical methods for estimating $R_{e f f}(t)$ have many shortcomings due to the characteristics of SARSCoV-2 transmission, namely silent transmission and major variation in the time to case reporting due to lack of timely or appropriate testing (Gostic et al., 2020). As such, an original mechanistic method was used based on a stochastic model (Figure A1 and Eqs. A1-A3, see online supplementary material) with timevarying parameters (Cazelles et al., 2018) and inferred with a Bayesian method using hospital data (Cazelles et al., 2021). Daily mobility data provided by Google were used, which are a proxy for real-time trends in movement patterns and human behaviour (Google, 2020). Google mobility data measure the percentage of change relative to pre-pandemic baseline mobility, and measure visits to and time spent in several areas: retail and recreation locations, supermarkets and pharmacies, public transport hubs, workplaces and at home.

This analysis revealed that trends in transmission estimated from well-documented hospital data correlated strongly with trends in mobility patterns within and between the first two waves of the COVID-19 pandemic for retail/recreation mobility, as well as public transport mobility. $R_{\text {eff }}(t)$ increased and oscillated in parallel with mobility; correlation between 15 May and 15 October 2020 was highly significant, with Pearson's correlation coefficient $>0.5$ (Figure 1). These relationships were particularly strong for retail/ recreation mobility and public transport mobility. In addition, cross-correlation functions for these datasets were computed, and revealed that correlations were maximal for a delay between 0 and 10 days, and this delay varied depending on the region (Figures A2 and $A 3$, see online supplementary material).

Earlier findings showed that mobility restriction can reduce the transmission of infection dramatically, and the present results confirmed that the reverse is also true; that is, increased mobility following the relaxation of lockdown rules can lead to an increase in transmission. This suggests the importance of tracking changes in mobility data at different spatial resolutions to anticipate future changes in the transmission of SARS-CoV-2 and guide public health policy.

The strong relationship between retail/recreational mobility and increased transmissibility suggests that a return to baseline human activity poses a significant risk of increased infection. Moreover, even if a direct causal link cannot be drawn, this strong correlation indicates that an increase in retail/recreation mobility promotes high-intensity contact between people and interaction between people of different households, resulting in increased transmission of SARS-CoV-2. This strong relationship is in agreement with the findings of Chang et al. (2021), who demonstrated that mobility network models can inform the reopening of society.

These findings illustrate the importance of mobility at defined regional levels, and, perhaps more importantly, show that public transport mobility and retail/recreation mobility are more strongly correlated with transmission than other forms of mobility. These findings will aid the refinement of possible lockdown policies and mitigation measures in the future while awaiting the 'roll out' of vaccines.

\section{Funding}

BC and BR are supported, in part, by Grant ANR Flash Covid-19 from Agence Nationale de la Recherche (DigEpi).

\section{Ethical approval}

Not required.

\section{Conflict of interest}

None declared. 


\section{Appendix A. Supplementary data}

Supplementary data associated with this article can be found, in the online version, at https://doi.org/10.1016/j.ijid.2021.01.067.

\section{References}

Badr HS, Du H, Marshall M, Dong E, Squire MM, Gardner LM. Association between mobility patterns and COVID-19 transmission in the USA: a mathematical modelling study. Lancet Infect Dis 2020;20:1247-54.

Cazelles B, Champagne C, Dureau J. Accounting for non-stationarity in epidemiology by embedding time-varying parameters in stochastic models. PLoS Comput Biol 2018; 14:e1006211.

Cazelles B, Nguyen Van Yen B, Champagne C, Comiskey C. Dynamics of the COVID-19 epidemic in Ireland under mitigation 2021. BMC Infect Dis 2021; , doi:http://dx. doi.org/10.21203/rs.3.rs-143697/v1.

Chang S, Pierson E, Koh PW, Gerardin J, Redbird B, Grusky D, et al. Mobility network models of COVID-19 explain inequities and inform reopening. Nature 2021;589:82-7.
Google. Google COVID-19 Community Mobility Reports. Mountain View, CA: Google; 2020 Available at: https://www.google.com/covid19/mobility/ [accessed 30.11.20].

Gostic KM, McGough L, Baskerville EB, Abbott S, Joshi K, Tedijanto C, et al. Practical considerations for measuring the effective reproductive number. PLoS Comput Biol 2020;16:e1008409.

Kraemer MUG, Yang CH, Gutierrez B, Wu CH, Klein B, Pigott DM, et al. The effect of human mobility and control measures on the COVID-19 epidemic in China. Science 2020;368:493-7.

Lemaitre JC, Perez-Saez J, Azman A, Rinaldo A, Fellay J. Assessing the impact of nonpharmaceutical interventions on SARS-CoV-2 transmission in Switzerland. Swiss Med Wkly 2020;150:w20295.

Park SW, Sun K, Viboud C, Grenfell BT, Dushoff J. Potential roles of social distancing in mitigating the spread of coronavirus disease 2019 (COVID-19) in South Korea. Emerg Infect Dis 2020;26:2697-700.

Tizzoni M, Bajardi P, Decuyper A, Kon Kam King G, Schneider CM, Blondel V, et al. On the use of human mobility proxies for modeling epidemics. PLoS Comput Biol 2014; 10:e1003716. 